\title{
Correction to: Early-life gut microbiota and neurodevelopment in preterm infants: any role for Bifidobacterium?
}

\author{
Isadora Beghetti ${ }^{1,5} \cdot$ Monica Barone $^{2} \cdot$ Silvia Turroni $^{3} \odot \cdot$ Elena Biagi ${ }^{3} \cdot$ Alessandra Sansavini $^{4} \cdot$ Patrizia Brigidi $^{2}$. \\ Luigi Corvaglia $^{1,5} \cdot$ Arianna Aceti $^{1,5}$
}

Published online: 13 January 2022

○) Springer-Verlag GmbH Germany, part of Springer Nature 2022

\section{Correction to: European Journal of Pediatrics https://doi.org/10.1007/s00431-021-04327-1}

In the original published version of the above article contained an error, the affiliation "Microbiomics Unit, Department of Medical and Surgical Sciences, University of Bologna, 40,138 Bologna, Italy" should have been removed for the following authors: Isadora Beghetti, Luigi Corvaglia and Arianna Aceti. The affiliation section has been updated.

The original article has been corrected.

Publisher's Note Springer Nature remains neutral with regard to jurisdictional claims in published maps and institutional affiliations.

The original article can be found online at https://doi.org/10.1007/ s00431-022-04382-2.

Silvia Turroni

silvia.turroni@unibo.it

Isadora Beghetti

isadora.beghetti@studio.unibo.it

Monica Barone

monica.barone@unibo.it

Elena Biagi

elena.biagi@unibo.it

Alessandra Sansavini

alessandra.sansavini@unibo.it

Patrizia Brigidi

patrizia.brigidi@unibo.it

Luigi Corvaglia

luigi.corvaglia@unibo.it
Arianna Aceti

arianna.aceti2@unibo.it

1 Neonatal Intensive Care Unit, IRCCS Azienda OspedalieroUniversitaria Bologna, Bologna 40138, Italy

2 Microbiomics Unit, Department of Medical and Surgical Sciences, University of Bologna, Bologna 40138, Italy

3 Unit of Microbiome Science and Biotechnology, Department of Pharmacy and Biotechnology, University of Bologna, Bologna 40126, Italy

4 Department of Psychology "Renzo Canestrari”, University of Bologna, Bologna 40127, Italy

5 Department of Medical and Surgical Sciences, University of Bologna, Bologna 40138, Italy 